\title{
Rezensionen / Reviews
}

Bitbol, Michel/Gayon, Jean, Hg., 2006. L'épistémologie française, 1830-1970. Paris: Presses Universitaires de France, $501 \mathrm{~S}$. Braunstein, Jean-François, Hg., 2007. Canguilhem. Histoire des sciences et politique du vivant. Paris: Presses Universitaires de France, $161 \mathrm{~S}$.

Stellt man die beiden vorliegenden Sammelbände in den Zusammenhang jüngst wieder explizit werdender Diskussionen zur Epistemologie naturwissenschaftlicher Erkenntnis, zum Beispiel zur historischen Epistemologie, leisten sie dazu unterschiedliche Beiträge. Während der von Michel Bitbol und Jean Gayon herausgegebene Band historisch angelegt ist - der Gegenstand, die französische Epistemologie, wird in seiner Geschichte konzipiert -, liegen mit der Aufsatzsammlung zu Georges Canguilhem Versuche eines produktiven Weiterdenkens von dessen epistemologischen Vorschlägen vor.

Die 21 Aufsätze des Buchs zur französischen Epistemologie dokumentieren für einen Untersuchungszeitraum, der mit dem Erscheinen von Auguste Comtes Cours de philosophie positive beginnt und mit der Emeritierung George Canguilhems endet, eine in Frankreich besonders intensive Beschäftigung mit den Wissenschaften. Epistemologien der Physik, Chemie und Biologie sind ebenso vertreten wie die Philosophie der Mathematik und die Logik. Die Autorinnen und Autoren befragen die bearbeiteten Originaltexte und historischen Zusammenhänge vorwiegend im Hinblick auf wissenschaftsphilosophische Problematiken. In einigen Beiträgen wird auf konzeptuell-theoretische Wechselwirkungen und explizite Auseinandersetzungen französischer Philosophen mit Diskussionskontexten wie Logischem Empirismus, analytischer Philosophie und Postpositivismus eingegangen. Sie sind der Schwerpunkt des ersten ("Traditions de pensée et institutions“) von insgesamt zwei Teilen und weisen die im Buch präsentierte Epistemologie als aus verstreuten Fasern konstituiert auf, die weit über die Grenzen des Französischsprachigen hinausreichen. So verdeutlichen sie, was für die Konzeption des Buches insgesamt gilt: Der Sammelband favorisiert nicht eindeutige Ursprünge oder eine kohärente Tradition „französischen“ Den- 
kens, sondern betont dessen Zerklüftung. Eine deutlichere Kenntlichmachung dieser Forschungsperspektive im Titel wäre von Vorteil gewesen. Als einzig durchgängiges Merkmal der vorgestellten Ansätze heben die Herausgeber ein eng an den Naturwissenschaften orientiertes Denken heraus, welches sich polemisch von allgemeiner gefassten Erkenntnistheorien - wie sie etwa mit dem englischen Term "epistemology“ aufgerufen werden - absetzt. Im umfassenderen zweiten Teil des Bandes sind vorwiegend Studien zu Konzepten und Philosophien herausragender Vertreter der französischen Wissenschaftsphilosophie versammelt (zum Beispiel Henri Poincaré, Emile Meyerson, Alexandre Koyré, Jean Cavaillès). Aber auch Autoren, denen bisher - zumindest außerhalb Frankreichs - wenig Aufmerksamkeit entgegengebracht wurde, werden behandelt (so etwa Jean Nicod, Jacques Herbrand oder Jean-Louis Destouches).

Die Artikel eines („Philosophie générale des sciences“) der insgesamt sieben Unterabschnitte haben für die im Buch präsentierte Auffassung von Epistemologie eine strategische Bedeutung. Genannt sei die Arbeit von Laurent Clauzade, der im Werk Auguste Comtes Elemente nachweist, die häufig vermittelt über die Namen Gaston Bachelard und Georges Canguilhem mit der „französischen Tradition“ der Epistemologie assoziiert werden (beispielsweise eine von der Gegenwart beurteilte Wissenschaftsgeschichte oder die Möglichkeit partikularer Wissenschaftsphilosophien). In derselben Rubrik zeigt Jean-Claude Pariente, wie Augustin Cournot im 19. Jahrhundert auf der Grundlage des Heliozentrismus eine Inkompatibilität von Kants Erkenntnistheorie mit der Objektivität und dem Fortschritt wissenschaftlichen Wissens feststellt. Das Nachdenken zu den Lebenswissenschaften wird ebenfalls als bestimmender Faktor der französischen Epistemologie ausgewiesen, denn der Artikel zu Claude Bernard (Gayon) erscheint in einer Reihe mit Comte, Cournot und Bachelard. Dem trägt außerdem der Abschnitt „Épistemologie des sciences de la vie et de la médecine“ mit Arbeiten zum Konzept der Individualität in der Biologiephilosophie Canguilhems (Gayon) und zur Medizinphilosophie Félix Ravaissons (Bitbol-Hespériès) Rechnung. Die vier unter „Positivisme“ gruppierten Arbeiten stellen durchweg Bezüge zur sogenannten analytischen Tradition der Wissenschaftsphilosophie her. Anastasios Brenner geht es um den neuen Positivismus in Frankreich ab 1900. Er kontextualisiert Pierre Duhems Holismus und Kritik des Induktionismus und liefert damit eine breitere Basis für die Untersuchung der Rückgriffe des Logischen Empirismus auf den Konventionalismus Poincarés. Antonia Soulez analysiert die Rezeptionsschwierigkeiten des Logischen Empirismus in Frankreich und macht dafür den programmatischen Stil Otto Neuraths sowie eine spezifische französische Auffassung des Verhältnisses von Logik und Sprache verantwortlich. Sandra Laugier unterstreicht die Kompatibilität der Philosophie Émile Meyersons mit einem „epistemologischen Holismus“ im Sinne der „Quine-Duhem“-These. 
Die lehrreichen Studien des Bandes knüpfen überwiegend an aktuelle Forschungsfragen an und setzen von daher einige wissenschaftsphilosophische Kenntnisse voraus, können aber durchaus als Einführungen gelesen werden. Ein Namensregister ist angefügt. Dem von den Herausgebern formulierten Anspruch, zugleich die verschiedenartige Zusammengesetztheit der französischen Epistemologie und ihre Synthesen von sonst getrennt erwogenen theoretischen Fragestellungen aufzuweisen, wird der Band mehr als gerecht.

Canguilhem. Histoire des sciences et politique du vivant setzt dort an, wo die Herausgeber von Lépistemologie française, 1830-1970 ihren thematischen Schlussstrich ziehen. Die Konzepte eines für die französische Epistemologie wichtigen Autors werden auf ihre Ergiebigkeit für eine Kritik der Psychologie, der Biomedizin und der Neurowissenschaften hin befragt. Die Applikationen auf aktuelle und historische Fälle sind dabei weder im Aufbau des Bandes noch in den einzelnen Aufsätzen von historischen Untersuchungen zu Werk und Biographie separiert, anders als zum Beispiel in dem von Borck/ Hess/Schmidgen 2005 herausgegebenen Band Maß und Eigensinn. Studien im Anschluß an Georges Canguilhem. Die resultierenden Synthesen demonstrieren somit ein Weiterdenken Canguilhems zugleich als eine Vertiefung von dessen Konzepten. Vereinzelt setzen Beiträge aber dadurch jene Tendenz der Hommagen an Canguilhem fort, die im Vorwort für überwunden erklärt wird. Als das eigentliche Medium der Kritiken Canguilhems stellt sich die Philosophie heraus, sei es, dass sie als „biomedizinische Philosophie“ (Dagognet), „philosophisches Engagement auf dem Feld der Medizin“ (Debru), vitaler Rationalismus (Dagognet) oder als umfassende Kritik der philosophischen Tradition Descartes' (Hacking) diskutiert werden. Eine für das kritische Studium der Wissenschaften in den letzten Jahrzehnten häufig zurückgewiesene Bedeutung der Philosophie betonen die Autoren im Ganzen somit am Modell der Canguilhem'schen Studien neu. Dass der Titel die Ubiquität des „Philosophischen“ im Band nicht artikuliert, verwundert.

Der Verknüpfung der fundamentalen Kritik behaviouristischer Psychologie einerseits und deterministischer und mechanistischer Konzeptionen des Milieus andererseits in Canguilhems Werk geht Jean-François Braunstein nach und betont, dass Canguilhem nicht bei ethischen Argumenten stehen bleibt, sondern wissenschaftliche Interventionen vornimmt. Claude Debru prüft Canguilhems Erwägungen zur Medizin als Technik auf eine medizinische Ethik hin. Er insistiert auf ihre Brauchbarkeit angesichts heutiger biomedizinischer Praktiken und gesundheitspolitischer Fragen zu Therapie und Pflege. Ian Hacking arbeitet in seinem Essay zugleich die Relevanz von Canguilhems Technikphilosophie für aktuelle Naturwissenschafts- und Technikstudien und die Durchschlagskraft von Canguilhems philosophischen Revisionen des Descartes'schen Dualismus heraus. Die Grenzziehungen zwischen 
Maschinen und Organismen bei der Erfindung des computergestützten BioFeedbacks in den 1960er Jahren werden diskutiert sowie Donna Haraways und Andrew Pickerings Auffassungen des „Cyborgs“. Arlid Utaker knüpft an Canguilhems prinzipielle Unterscheidung der Gegenstände „Denken“ und "Gehirn“ an und stützt sich dabei auf Parallelen mit dem Spätwerk Ludwig Wittgensteins. Während das Gehirn einer Erforschung durch empirische Wissenschaften zugänglich ist, bleibt das Denken ein Sujet der Philosophie. Mit dieser Demarkation ergeben sich zum Beispiel konkrete Grenzen neurowissenschaftlicher Aussagen. In Stil und Umfang sind die Beiträge recht heterogen, was durch ihre geringe Anzahl ins Gewicht fällt. Der Sammlung ist keine explizite integrierende Problemstellung zugrunde gelegt, obwohl das präsentierte Material durch vielfache Kohärenzen Ansatzpunkte dafür geboten hätte.

Mit den beiden Büchern im Ensemble zeichnet sich eine Auffassung von Epistemologie ab, bei der die zeitgenössischen Naturwissenschaften, ihre Geschichte und ihre Philosophie eng aufeinander verwiesen sind. Dass dieses wechselseitige Verhältnis aber ganz unterschiedlich ausgelotet werden kann, dafür wird ein breites Spektrum an Beispielen zur Verfügung gestellt.

Sandra Pravica (Berlin)

Park, Katharine/Daston, Lorraine, Hg., 2006. Early Modern Science. Cambridge: Cambridge University Press (=The Cambridge History of Science, 3), 894 S., $96.00 €$, ISBN-13: 978-0521572446.

Philosophie und Naturwissenschaften waren in der frühen Neuzeit noch ungeschieden, die meisten Naturforscher, die wir heute als Mediziner, Chemiker, Physiker, Mathematiker oder Biologen bezeichnen, nannten sich Philosophen. Deshalb gibt es bei historischen Untersuchungen zur frühen Neuzeit eine große Schnittmenge von philosophie- und wissenschaftsgeschichtlich relevanten Themen und Akteuren. Zur Philosophiegeschichte der frühen Neuzeit liegt bereits aus der Cambridge-Serie eine Reihe größerer Überblicksdarstellungen vor, die jüngste darunter ist die Cambridge History of 17th-Century Philosophy (1998). Auch die von 1998-2001 erschienenen vier Doppelbände der Philosophie des 17. Jahrhunderts aus der von Friedrich Ueberweg begründeten Reihe Grundrisse der Philosophie sind eine unverzichtbare Arbeitsgrundlage. Der hier vorzustellende Band ist trotz seiner knapp 900 Seiten, auf denen die Wissenschaften der frühen Neuzeit für den Zeitraum von circa 1490-1730 dargestellt werden, im Vergleich dazu kompakt ausgefallen. Thematische Überschneidungen mit den Vorgängerbänden waren unvermeidbar, erkennbar ist nun jedoch der Versuch einer stärkeren Ausrichtung auf die neuere Wissenschaftsgeschichtsschreibung angloamerikanischer Prägung. Unter Verzicht auf ältere historiographische Schemata, 
für die die Annahme einer wissenschaftlichen Revolution in der frühen Neuzeit zentraler Ausgangspunkt der Darstellung ist, wird in diesem Band ein buntes, vielschichtiges Bild der Ungleichzeitigkeiten und Verflechtungen unterschiedlichster Wissens- und Wissenschaftsformen dieses Zeitraums gezeichnet. Die Herausgeberinnen versuchen traditionelle historiographische Konventionen aufzubrechen, indem sie Beiträge versammeln, die Themen und Entwicklungen frühneuzeitlicher Wissenschaft aus ganz unterschiedlichen Perspektiven beleuchten. Obgleich die wissenschaftliche Revolution als Erklärungsmuster verabschiedet wird, bleibt indes die ebenso problematische Kategorie des „Neuen“ als Leitmotiv in Kraft.

Der Band ist in vier Hauptteile gegliedert, deren Beiträge jeweils wechselseitig aufeinander bezogen gelesen werden sollen. Werden in einem Teil wissenschaftliche Innovationen und Konzeptionen vorgestellt, ist in einem anderen nachzulesen, an welchen Orten dieses Wissen von welchen sozialen Gruppen wie erzeugt wurde, wie dieses Wissen dann durch welche Medien wann, wie und wo verbreitet und zu welchen praktischen Zwecken es nutzbar gemacht beziehungsweise erst hervorgebracht wurde. Die Beiträge des ersten, mit „The New Nature“ überschriebenen Teils führen daher zunächst das Spektrum frühneuzeitlicher wissenschaftlicher Erklärungsformen vor. Die Studien im zweiten Teil („Personae and Sites of Natural Knowledge“) zeigen dann, wer wo welches Wissen erzeugte. Im dritten Teil werden Einteilungen und Ordnungssysteme in frühneuzeitlicher Theoriebildung und Forschungspraxis vorgestellt („Dividing the Study of Nature“), und schließlich loten die Autoren im letzten Teil unter dem Stichwort „Cultural Meanings of Natural Knowledge“ die Interaktionen von Naturphilosophie, Religion, Kunst und Literatur aus.

Geboten wird so in dichter Form eine Fülle von Sachinformationen zu sehr vielen Themenfeldern, deren breite Streuung sich darin dokumentiert, dass Artikel zu Astronomie, Chemie, Akustik, Optik oder Reiner Mathematik neben Beiträgen zur Militärtechnik, zur Rolle von Caféhäusern und Marktplätzen, zu Reise- und Briefnetzwerken, Genderfragen, Magie, Astrologie und Kosmographie stehen. Bei 33 hochkarätigen Einzelbeiträgen aus der Feder namhafter Autorinnen und Autoren wundert es nicht, dass nicht alle gleichermaßen den Vorstellungen der Herausgeberinnen folgen. Diese weisen in ihrer Einleitung darauf hin, dass der Mythos der wissenschaftlichen Revolution sich bislang als unbesiegbar erweist, da das heutige Verständnis der kulturellen Überlegenheit und Modernität des Westens zu eng mit ihm verknüpft sei. Auch der vorliegende Band wird immer wieder von diesem Mythos eingeholt. Dies zeigt sich etwa darin, dass mit den mathematischen Disziplinen verbundene Themen (Optik, Astronomie), die das Rückgrat der älteren Historiographie bildeten, gut repräsentiert bleiben, während für die frühe Neuzeit wichtige Experimentierfelder für nichtmathematische Methoden, wie etwa Meteorologie und Embryologie nur am Rande gestreift wer- 
den. Im Zweifel werden dann doch eher die Lehrbuchtexte zitiert, wie etwa Bacons Novum Organum und nicht seine damals wirkmächtigere Schrift Sylva Sylvarum. Auch in der geographischen Vermessung der Frühen Neuzeit dominiert weitgehend der alte Kanon: Nord- und Osteuropa, Spanien und Portugal sind stark vernachlässigt, Deutschland und Frankreich im Verhältnis zu England unterrepräsentiert.

Mit der Aufnahme von Artikeln zu Kunst und Literatur soll die Unhintergehbarkeit rhetorischer und ästhetischer Formen für viele Wissensbereiche klargemacht werden. Die Herausgeberinnen betonen sogar, dass diese Beiträge nicht als bloße Ergänzungen der Wissenschaftsgeschichte um weitere Aspekte („science plus x“) zu verstehen seien, da in der frühen Neuzeit Kunst und Wissenschaft untrennbar miteinander verwoben waren. Angesichts dessen wäre es sinnvoll gewesen, den vierten Teil nicht zu separieren, vielmehr hätten die in ihm vorgeführten Wechselwirkungen zur Voraussetzung für die Rekonstruktion auch der vermeintlich eher wissenschaftlichen Themen gemacht werden können. So hätten etwa in den Beiträgen zur Mechanik, zu den Beweisverfahren, zu den Erfahrungskonzepten das Interagieren ästhetischer, rhetorischer und traditionell demonstrativer Verfahren vorgeführt oder die Wechselwirkung in der Ausbildung neuer Semantiken und Werte konkret aufgezeigt werden können. Eine solche Verschränkung gelingt eher selten, wie etwa in Richard W. Serjeantsons Untersuchung des Zusammenspiels von „Proof and Persuasion“ in frühneuzeitlichen Überzeugungsstrategien.

Grund dafür ist, dass der Band weniger als ein Handbuch für die Forschung, sondern mehr als ein Kompendium konzipiert ist, mit dem wichtige Forschungserträge der vergangenen Jahrzehnte in konziser und für die Lehre praktikabler Form schnell greifbar gemacht werden sollen. So resümieren unter anderem Dan Garber über das Verhältnis von Metaphysik und Physik in der frühen Neuzeit, Londa Schiebinger über naturforschende Frauen, Adrian Johns über das Buchwesen, Jim Bennett zur Rolle wissenschaftlicher Instrumente oder Brian Copenhaver zur Magie ihre eigenen und im Kern bekannten Forschungspositionen. In diesem Zusammenhang vermisst man jedoch die Einbeziehung von Forschungskontroversen. In vielen Themenfeldern der Frühneuzeitforschung gibt es keinen Konsens, zuweilen sogar regelrechte Lager, zum Beispiel im Hinblick auf die von Garber vertretene Einschätzung des damaligen Verhältnisses von Metaphysik und empirischer Forschungspraxis. Der Heterogenität der frühen Neuzeit entspricht auch eine große Vielfalt der Rekonstruktionen und verschiedenen Auslegungstraditionen. Trotz vieler Differenzen im Detail versuchen die Autorinnen und Autoren des Bandes eine neue Wissenschaftsgeschichtsschreibung als kohärente Kollektivanstrengung zu präsentieren und Spannungen eher zu kaschieren. Die Querverweise innerhalb des Bandes dienen entweder der wechselseitigen Bestätigung oder der Delegierung von Aufgaben, nicht aber 
der kritischen Auseinandersetzung mit den Positionen der Kollegen - so entsteht der Eindruck weitgehender Einmütigkeit.

Von einem Beitrag in einem allgemein angelegten wissenschaftshistorischen Kompendium sollte man jedoch erwarten, dass in ihm auch andere Positionen als die, für die der jeweilige Autor beziehungsweise die jeweilige Autorin einsteht, dargestellt werden. So werden etwa die von Peter Dear zu erklärenden "Meanings of Experience“ primär anhand englischer Beispiele erläutert und dennoch als exemplarisch für die frühe Neuzeit behauptet, obgleich etwa im deutschen oder französischen Kontext andere Semantiken und Praktiken zu verzeichnen sind. Zwar werden solche Einseitigkeiten durch herausragende Einzelbeiträge ausgeglichen, insgesamt dominiert jedoch ein starker Anglozentrismus. Deutlich wird diese Einschränkung auch in der Darstellung der frühneuzeitlichen Literaturgeschichte, die aus der Feder einer Anglistin mit dem Schwerpunkt Reiseliteratur stammt: Mary Baine Campbell unternimmt eine tour d'horizon durch die englische Literaturgeschichte, garniert mit einigen Beispielen aus der Höhenkammliteratur anderer Nationen - sofern diese auf Englisch vorliegen. Hingegen zeigt gleich der nächste Artikel, dass es auch anders geht: Carmen Niekrasz und Claudia Swan gelingt es, auf wenigen Seiten eine exzellente problemorientierte Übersicht der Wechselwirkungen von Kunst und Wissenschaft in Europa zu geben.

Es fällt auf, dass fast alle Autoren an amerikanischen oder britischen Universitäten angestellt sind, und es fragt sich, welches Statement hinter dieser Herausgeberentscheidung steckt? Die meisten Beiträger zitieren fast nur Quellen und Sekundärliteratur, die in englischer Sprache vorliegen, offenbar in der Annahme, dass die frühe Neuzeit lediglich auf der Basis von ins Englische übersetzten Primär- und Sekundärtexten zu rekonstruieren wäre. $\mathrm{Zu}$ viele Beiträge bewegen sich dadurch innerhalb eines künstlich geschaffenen, (nahezu) rein englischsprachigen Referenzsystems.

Claus Zittel (Florenz/Olsztyn/Frankfurt am Main)

Hausdorff, Felix, 2008. Gesammelte Werke, Band III: Mengenlehre
(1927, 1935). Deskriptive Mengenlehre und Topologie. Herausgegeben von Ulrich Felgner, Horst Herrlich, Mirek Hušek, Vladimir Kanovei, Peter Koepke, Gerhard Preuß, Walter Purkert und Erhard Scholz. Berlin u.a.: Springer, xxii + 1005 S., geb. $99.95 €$, ISBN-13: 978-3-540-76806-7.

Mit diesem Buch ist jetzt der seit 2001 fünfte von neun Bänden der Werkedition des bedeutenden deutschen Mathematikers Felix Hausdorff (18681942) erschienen, über die an dieser Stelle schon mehrfach berichtet wurde (NTM 11 (2003): 198f., 12 (2004): 124, 14 (2006): 60f., 15 (2007): 307-309). Inhaltlich schließt die Publikation eng an den 2002 veröffentlichten Band II 
an, in dem Hausdorffs wohl wichtigstes Werk, die Grundzüge der Mengenlehre (1914), ediert ist. Der eigentliche Grund, nun auch die 1927 erschienene zweite Auflage der Grundzüge in dieser Werkausgabe zu veröffentlichen und sorgfältig zu kommentieren (siehe S. 42-351, in der wenig veränderten, im Wesentlichen um ein Kapitel erweiterten Form der 3. Auflage von 1935), liegt in der ungewöhnlichen Geschichte des von Hausdorff nur noch „Mengenlehre" genannten Werkes. Purkert und Kanovei machen in ihrer instruktiven „Historischen Einführung“ (S. 1-40) deutlich, dass äußere, vom Verlag gestellte Bedingungen, die eine starke Kürzung des Umfangs veranlassten, mit den inzwischen stark veränderten Forschungsinteressen des nahezu sechzigjährigen Hausdorff zusammentrafen, so dass es sich bei der 2. Auflage eigentlich um ein völlig neues Buch handelte. Während die Grundzüge von 1914 ihre Hauptwirkung in der mengentheoretischen Topologie entfalteten, rekapitulierte die "Mengenlehre“ die inzwischen erfolgte Entwicklung eines anderen Zweiges der Mengenlehre, nämlich die der sogenannten deskriptiven Mengenlehre. Sie nahm ihren Ausgangspunkt in französischen Arbeiten (René Baire und die Klassifikation reeller Funktionen) um 1900 und wurde in den folgenden Jahrzehnten vor allem von russischen und polnischen Mathematikern wie Nikolaj N. Lusin und Wacław Sierpiński, aber auch von Hausdorff selbst (Lösung des Kontinuumproblems für Borelmengen 1916) entwickelt. Es ist so nicht zufällig, dass in den veröffentlichten und unveröffentlichten Beiträgen Hausdorffs über die deskriptive Mengenlehre, die in dem vorliegenden Band ediert und kommentiert werden, der Bezug zu und die Kommunikation mit russischen und polnischen Mathematikern einen wesentlichen Platz beansprucht. Der Edition gelingt es, die Bedeutung der veröffentlichten Beiträge Hausdorffs für die moderne, von Begriffen wie „polnische Räume“ geprägte und in Disziplinen wie Funktionalanalysis, Limitierungstheorie und Nichtstandard-Analysis hineinreichende deskriptive Mengenlehre deutlich zu machen. Dasselbe betrifft den Wiederabdruck zweier wichtiger Arbeiten von Hausdorff über metrische Räume und ihre Abbildungen (1924 und 1930).

Was die unveröffentlichten Beiträge Hausdorffs zur deskriptiven Mengenlehre betrifft, so musste eine Auswahl aus über tausend Blatt des Nachlasses getroffen werden. Hausdorff selbst hat diese Notizen weitgehend zum eigenen Verständnis beim Studium einschlägiger Arbeiten angefertigt. Die Herausgeber weisen auf das in diesen Aufzeichnungen liegende Potential für moderne Forschungen hin, deuten aber auch an (S. 31, 845), dass die Zeitumstände und die antisemitische Politik der 1930er Jahre die Publikationsmöglichkeiten für Hausdorff eingeschränkt haben.

Das letzte Viertel des Bandes ist Hausdorffs unveröffentlichten Arbeiten zur Topologie gewidmet, geordnet nach Manuskripten zur allgemeinen (mengentheoretischen) Topologie und zur kombinatorischen oder algebraischen Topologie, mit der sich Hausdorff erst relativ spät, seit dem Ende 
der 1920er Jahre beschäftigte. Ihr hat er eine einzige Vorlesung (im Sommersemester 1933) gewidmet, die bemerkenswert modern war und deren Skript aus dem Nachlass ebenfalls abgedruckt ist (S. 893-953). Dieser Abschnitt der Edition enthält auch vier weitere Essays zur historischen Einordnung von Hausdorffs Arbeiten, von denen insbesondere die beiden längsten über seine Abhandlungen zum Kurvenbegriff (S. 798-825, von Herrlich, Hušek, Preuß), und „Hausdorffs Blick auf die entstehende algebraische Topologie“ (S. 865-892, Scholz) eine selbständige Bedeutung beanspruchen können.

Alle Beiträge Hausdorffs und die meisten Kommentare sind auf Deutsch publiziert. Eine Datierung der Arbeiten auch im Inhaltsverzeichnis (soweit möglich) wäre wünschenswert gewesen; die Abschnittsüberschriften des Inhaltsverzeichnisses werden am entsprechenden Ort hinten nicht wiederholt. Abgesehen von diesen kleinen Schönheitsfehlern erfüllt das Buch aber erneut den von den bisherigen Bänden befolgten hohen editorischen Standard. Es enthält sorgfältige Literaturangaben, Personen- und Sachregister und - wie in jedem Band - das komplette Schriftenverzeichnis von Felix Hausdorff.

Reinhard Siegmund-Schultze (Kristiansand)

\section{Meier, Marietta/Bernet, Brigitta/Dubach, Roswitha/Germann, Urs, 2007. Zwang zur Ordnung. Psychiatrie im Kanton Zürich, 1870-1970. Zürich: Chronos Verlag, 280 S., CHF 48.00, ISBN-13: 978- 3034007856 .}

Mit diesem Sammelband ist eine neue und insgesamt gut gelungene Geschichte des psychiatrischen Zwangs erschienen. In Anlehnung an Robert Castel wird die psychiatrische Ordnung als „Bindeglied zwischen der gesellschaftlichen Ordnung und der (gestörten) Ordnung des Selbst" (S. 10) betrachtet. Ziel der Untersuchung ist es, für diese drei Ordnungsdimensionen (Institution, Selbst, Gesellschaft) die historischen Spannungsfelder von jeweils Zwang und Freiheit, Autonomie und Unterwerfung, sozialer Integration und gesellschaftlichem Ausschluss auszuloten. Es geht vor allem darum, die Vieldeutigkeit des Zwangs sowohl innerhalb als auch zwischen diesen Ordnungsdimensionen $\mathrm{zu}$ thematisieren und dadurch ihre respektiven Übereinstimmungen und Dissonanzen zu erfassen. Grundsätzlich wird dabei die „Sicht der PatientInnen“ privilegiert, um „die medizinische, anstaltstechnische oder soziologische Sicht" (S. 44) zu konterkarieren. In Kombination mit einem sozialwissenschaftlich-quantitativen Zugriff auf die Patientenakten der Universitätsklinik Burghölzli und (ab 1913) ihrer Poliklinik sowie auf die der Pflegeanstalt Rheinau liefert dieser Ansatz wichtige und zum Teil überraschende Forschungsergebnisse.

In einem gemeinsam geschriebenen ersten Teil wird das institutionelle Setting erläutert und ein quantitativer Überblick geboten. Bereits hier ge- 
lingt es, neue Akzente in der Forschung zu setzen, indem überzogene sozialgeschichtliche Deutungsparadigmen zugunsten bislang unterrepräsentierter Geschlechterperspektiven zurückgedrängt werden. So hat die Autorengruppe eine statistisch signifikante Diskriminierung gesellschaftlicher Unterschichten beispielsweise nur in Bezug auf die Wahrscheinlichkeit von disziplinierenden Maßnahmen, nicht aber hinsichtlich der Einweisung oder der Diagnosenstellung feststellen können. Demgegenüber wird eine starke Geschlechterasymmetrie nachgewiesen, die sich unter anderem darin äußert, dass Frauen häufiger mit Schizophrenie diagnostiziert und zur Zielscheibe therapeutisch-disziplinierender Motive wurden.

Im zweiten Teil des Buches werden jeweils einzelne Themenkomplexe (Entmündigung, Sterilisation, Beschäftigungstherapie, Psychochirurgie) abgehandelt. Brigitta Bernets knüpft in ihrem Beitrag an eine alte Forschungstradition an, nach der die Psychiatrie als Spiegelbild bürgerlicher Wertvorstellungen gedeutet wird. Aus dieser Betrachtungsweise stellt sich die Psychiatriekritik um 1900 als „Effekt der staats- und wissenschaftspolitischen Neuausrichtung hin auf das Soziale“ (S. 152) dar. Als Antwort auf das aufkommende psychiatrische Expertenwissen einerseits und die Formierung einer sozialbehördlichen Interventionspolitik andererseits ist nach Bernet ein neuer, als „genossenschaftlich“ bezeichneter Subjektivitätstypus entstanden, der das alte „herrschaftliche Selbst“ immer mehr verdrängte. Und weil die Psychiatrie zur „Vertreterin und Vollstreckerin des ,sozialen Prinzips“ und somit ... zum Inbegriff des ,bürgerlichen Tods" um 1900“ wurde, geriet sie in die Kritik (S. 153). Leider wird die Zugänglichkeit dieses Kapitels durch den schwer zu durchdringenden Sprachstil der Autorin gemindert.

In ihrem Beitrag zur Sterilisationspraxis widerlegt Roswitha Dubach die sogenannte „Eugenik-Dominanztheorie“ (S. 158). Anhand der Akten der psychiatrischen Poliklinik und mit einer Gender-Fragestellung zeigt sie, dass die Sterilisationspolitik keine deutlich eugenisch motivierte Zwangspraxis darstellte, sondern viel stärker an die sozialen Komplexitäten der Abtreibungsfrage gekoppelt war. Damit kann sie (einmal mehr) die analytische Einseitigkeit der Raphael'schen These einer „Verwissenschaftlichung des Sozialen" aufzeigen. Viel eher könne man von einer ,Sozialisierung' medizinischer Maßnahmen sprechen, denn das Handeln psychiatrischer Experten in der Abtreibungs- und Sterilisationspraxis wurde in erster Linie nicht von medizinischen Kriterien, sondern sehr augenfällig von „gesellschaftlichen Normen- und Wertekomplexen, eugenischen Ordnungsvorstellungen - die ihrerseits stark mit sozialen Kriterien durchmischt waren - und aktuellen gesellschaftspolitischen Kontexten“ (S. 191) bestimmt.

Am konsequentesten wird das Gesamtkonzept des Bandes in den Beiträgen von Urs Germann und Marietta Meier umgesetzt. Anhand der drei Referenzordnungen untersucht Germann die Entwicklung der „aktiveren Krankenbehandlung" Hermann Simons. Eine Allianz von Arbeit und The- 
rapie führte die Zürcher Psychiatrie weg von einer Wachsaalpsychiatrie hin zu einer therapeutischen Praxis, in der versucht wurde, ein modernes Arbeitsethos nachzubilden. Meier untersucht in ihrem Beitrag zu psychochirurgischen Eingriffen die Wechselwirkung zwischen Diskurs und Praxis und unterstreicht dabei die Wichtigkeit der ,sozialen Heilung' als Referenzkategorie. Sie kann feststellen, dass Leukotomien meist bei Frauen, die als ,schwierige' Patienten galten, durchgeführt wurden und dass ihr postoperatives Sozialverhalten zum therapeutischen Erfolgskriterium geriet.

$\mathrm{Zu}$ Recht verweist Jakob Tanner in seinem Schlusswort auf die innovativen Aspekte dieses Bandes und lobt die Verabschiedung von der historiographischen Fixierung auf die ,große Einschließung. In der Tat befasst sich die neuere Historiographie zunehmend mit Fragen der institutionellen Ausdifferenzierung und der steigenden Durchlässigkeit von Handlungsfeldern beziehungsweise Akteursnetzwerken. Eine große Stärke des Bandes ist aber leider zugleich auch seine größte Schwäche: die quantifizierende Methode. Denn wie das Autorenteam selbst erkennt, büßt sie in der zweiten Hälfte des Untersuchungszeitraums ihre analytische Schärfe deutlich ein und ist nicht mehr in der Lage, die Komplexität der Handlungsmotive der Akteure zu erfassen. Diese Schallmauer sozialwissenschaftlichen Deutungsvermögens hinterlässt Fragen: Wie ist beispielsweise der auffallende Widerspruch zwischen der verschwindend geringen Bedeutung der Eugenik als statistisch-ermittelbares Handlungsmotiv einerseits und die ausgeprägt „sozialreformerisch-eugenische" Traditionslinie der Zürcher Schule andererseits zu erklären? Hinweise auf „ein Spannungsfeld verschiedener Ordnungsdimensionen" (S. 114) oder Tanners zwar nachvollziehbares (quodcumque in mundo est, non est in actis), aber dennoch gequältes Argument vom „methodologischen Dahinterismus“ (S. 294) können nicht befriedigen. Umso dankbarer ist man deshalb für die plastischen Narrative der Fallbeispiele, die die historisch-situative Vieldeutigkeit des psychiatrischen Zwangs in Zürich viel leistungsfähiger transportiert und das wackere Forschungsschiff NFP-51 vor dem stummen Untergang im Datenmeer gerettet haben.

Eric Engstrom (Berlin)

Hagner, Michael/Hörl, Erich, Hg., 2008. Transformation des Humanen. Beiträge zur Kulturgeschichte der Kybernetik. Frankfurt a. M.: Suhrkamp, brosch. 450 S., $14 €$, ISBN-13: 978-3518294482.

Pickering, Andrew, 2007. Kybernetik und Neue Ontologien. Aus dem Englischen von Gustav Roßler. Berlin: Merve, brosch. 184 S., $15 €$, ISBN-13: 978-3883962290.

In der Wissenschaftsgeschichte ist die Kybernetik aktueller denn je. Michael Hagner und Erich Hörl hat das veranlasst, ihrer Kulturgeschichte einen Sam- 
melband zu widmen. Auch Andrew Pickering geht in seiner jüngst erschienenen Aufsatzsammlung auf die Kybernetik ein und macht damit neugierig auf seine angekündigte Monographie zur Kybernetik.

Vor gerade einmal 60 Jahren versammelte Norbert Wiener eine interdisziplinäre Bewegung unter dem Begriff der Kybernetik. Das Duo Hagner/Hörl weiß aus der Kürze dieser Epoche intellektuelles Kapital zu schlagen. Ihre in der lesenswerten Einleitung formulierte These lautet: Was die Kybernetik an Versprechungen nicht einzulösen vermochte, betrifft uns heute genauso wie die Kontrollgesellschaft, die sie konkret umzusetzen geholfen hat. Doch nicht in allen Beiträgen ist es gleichermaßen gelungen, diese Fragestellung und einen zugegeben mitunter überladenen Gegenstand im Fokus zu behalten. Allein die schiere Fülle von mitunter auch konträren Perspektiven zeugt davon, dass von der Kybernetik nach wie vor eine diskursive Produktivität ausgeht. Werden Pickerings Essays zur Kybernetik hinzugezogen - die im Übrigen gut zu einem einzigen hätten verschmolzen werden können, um unnötige Redundanzen zu vermeiden -, dann ergibt sich eine beachtliche Liste an Themen, die sich unter dem Stichwort Kybernetik verbinden lassen. Pickering konzentriert sich auf die britischen Kybernetiker der ersten Stunde. Allen voran befreite der Psychiater Ross Ashby mit seinen Homöostaten die Maschinen davon, noch irgendetwas anderes zu produzieren als Erkenntnisgewinne in Form von Modellen, die nach Möglichkeit Strukturhomologien mit Gehirnen aufweisen sollten.

Im Band von Hagner und Hörl, in dem sich die Autoren und Autorinnen mit der Kybernetik zu Zeiten ihrer größten institutionellen Verbreitung auseinandersetzen, dominieren die Themen Kybernetisierung der menschlichen Ausbildung (Jürgen Oelkers, Urs Stäheli, Ulrich Bröckling und David Gugerli), spezifische Ästhetiken und Wahrnehmungsweisen der Kybernetik (Claus Pias, Christoph Asendorf und Cornelius Borck) sowie ihr Verhältnis zu benachbarten Disziplinen (Maria-Sibylla Lotter,Wolfgang Pircher und Jakob Tanner). Slava Gerovitch zeigt in seinem Beitrag anhand der wissenschaftlichen Laufbahn des Linguisten Roman Jakobson, wie die Kybernetik eine wissenschaftspolitische Dimension in der Sowjetunion eröffnete. Mit ihr klarzukommen konnte mitunter über "Sein oder Nichtsein“ entscheiden, während die Kybernetik im Westen eine Frage der Optionen blieb.

$\mathrm{Zu}$ kurz zu kommen droht allerdings in einigen Beiträgen die Frage, die der Titel „Transformation des Humanen“ aufwirft. Fraglich scheint, ob der von Michael Hagner in seinem Beitrag vertretene Ansatz, die Kybernetik vor dem Hintergrund der These Charles P. Snows von den zwei Kulturen zu diskutieren, hilft, ihren historischen Stellenwert zu bestimmen. Für Hagner ist dadurch vorgezeichnet, dass die Kybernetik letztlich „sang- und klanglos in den Kellerräumen der Wissenschaftsgeschichte" (S. 71) ihr Ende findet. Ein solches Fazit klingt weitaus abgeklärter als jene Aktualität der Kybernetik, von der der Klappentext kündet: „Wer über die anthropologischen 
Bedingungen der Gegenwart nachdenkt, wird nicht umhinkommen, sich mit der kybernetischen Transformation des Humanen zu befassen, deren Nachfahren wir sind." Hagner blendet aus, dass im Zentrum der Kybernetik nicht zwei „Wissenskulturen“ aufeinander prallten, sondern zwei Disziplinen, die zu den Naturwissenschaften zu zählen sind und die selbst schon vor Verständigungsproblemen standen. Die Rede ist einerseits von der Biologie, zu der hier noch die Physiologie und Medizin hinzuzuzählen wären, und andererseits von der angewandten Physik, vor allem in ihrer ingenieurmäßigen Ausrichtung auf die Elektro- und Regelungstechnik. So gesehen hat der Kern des kybernetischen Forschungsprogramms heute noch Bestand: die Identifizierung von operativen Modellen, um Komplexitäten von Organismen und Maschinen gleichermaßen zu erschließen und damit einen wechselseitigen Wissenstransfer zwischen Lebenswissenschaften und Informationstechnologien zu ermöglichen. Deshalb gab es das DFGSchwerpunktprogramm „Kybernetik“ ab 1965 und den Sonderforschungsbereich 50 „Kybernetik“ von 1969 bis 1983, und deshalb existiert das MaxPlanck-Institut für biologische Kybernetik heute noch. Werner Reichardts bahnbrechende Experimente über das Sehen bei Insekten führten zur Institutsgründung in den 1960er Jahren. Seine Forschung würde wohl heute noch mühelos jede beantragte Förderung erhalten - vorausgesetzt, auf die Verwendung des abgenutzten Stichworts „Kybernetik“ würde verzichtet und die neuesten bildgebenden und molekular biologischen Verfahren würden hinzugezogen werden.

Vor allem Erich Hörl stößt in seinem Beitrag zum harten historischen Kern der Kybernetik vor, indem er sich bei seinen Betrachtungen an den Neurophysiologen Warren McCulloch hält. Für diesen galt das Nervensystem als „logische Maschine par excellence“. Wie David Mindell in seiner großen Studie zum Stand der Regelungstechnik kurz vor dem Aufkommen der Kybernetik zeigen konnte, waren Techniken zur Regelung von Prozessen schon über zwei Jahrzehnte zusammengetragen worden, bevor man sich ihrer in der Kybernetik bediente. Was mit der Kybernetik aufkam, war ihre Anwendung auf die Lebenswissenschaften. Und weil die Kybernetik auf die Regelung von Kommunikation und Information hinauslief, schlug sie, wie Hörl in aufregender Weise begreiflich macht, durch bis zu den sogenannten Geisteswissenschaften. Aristoteles hat den Menschen als Lebewesen herausgehoben, das über Sprache verfügt. Die Kybernetik ließ Maschinen, Menschen und letztlich alle Lebewesen gleichermaßen in Kommunikationsmodelle aufgehen. Diese fundamentale Distinktionsaufhebung, so rekonstruiert Hörl, hat in ihrer ganzen Tragweite insbesondere einen fundamentalontologischen Sprachphilosophen in höchste Alarmbereitschaft versetzt: Martin Heidegger. Durch Hörl erfahren wir aber auch, dass und wie Heidegger sich das Feld der Kybernetik durch den Hegelianer und bekennenden Kybernetiker Gotthard Günther erschloss. 
Das abschließende Wort zur Kybernetik ist weder mit Hagners und Hörls noch mit Pickerings Buch geschrieben worden. Vielmehr eröffnen beide Bücher dort neue Horizonte, wo sie die Nachwirkungen der Kybernetik nicht nur zum Gegenstand ihrer Reflexion erheben, sondern auch in ihr neue epistemologische Möglichkeitsbedingungen erblicken. So empfiehlt auch Pickering die Auseinandersetzung mit der Kybernetik Historikerinnen und Historikern „als eine Art von Praxisfeld für unsere über-disziplinierten Phantasien" (S. 155).

Philipp von Hilgers (Berlin)

Heßler, Martina, 2007. Die kreative Stadt. Zur Neuerfindung eines Topos. Bielefeld: transcript (=Urban Studies), 360 S., $29.80 €$, ISBN-13: 978-3899427257.

Mit der Gründung sogenannter „Forschungsstädte“, „Technopolen“ oder "kreativer Städte“ hat sich seit den sechziger Jahren eine neue räumliche Organisationsform der naturwissenschaftlichen Forschung herausgebildet. Dabei handelt es sich um meist an der Peripherie großer Städte gelegene Komplexe von Forschungseinrichtungen, technologieorientierten Unternehmen und rudimentären Siedlungsstrukturen, die ein kreatives Umfeld für Forschung und technische Innovation bieten sollen. Drei solcher Orte, alle im Umfeld von München angesiedelt, stehen im Mittelpunkt der historischen Studie von Martina Heßler: das Atomforschungszentrum Garching, der Biotechnologie-Standort Martinsried sowie der Forschungskomplex für Mikroelektronik in Neuperlach.

Die Autorin, die ihr Buch explizit als einen Beitrag zum "spatial turn“ in den Geschichtswissenschaften begreift, macht anhand dieser drei Orte, gleichsam wie in einem Brennglas, das lokale Zusammenwirken einer Vielzahl gestaltender Prozesse sichtbar. Ihr gelingt damit nicht nur eine spannende Verbindung von Wissenschaftsgeschichte und Stadtgeschichte, sie zeigt auch, wie sich übergreifende gesellschaftliche Transformationsprozesse mit der sozialen, politischen und materiellen Dynamik konkreter Orte verzahnen. Thema der Studie sind die Programmatiken und Leitbilder, die dem Ausbau dieser Forschungsstätten zu Grunde lagen, die daraus resultierenden baulichen und sozialen Strukturen sowie die Reaktionen von Kommunalpolitikern und Bürgern, die diese Entwicklungen teils unterstützten, teils aber auch mit Protesten zu verhindern versuchten.

Wie die Autorin zeigt, ging es bei der Entwicklung der ersten Wissenschaftsstädte zunächst noch ausschließlich darum, die interdisziplinäre Kooperation im Bereich der Grundlagenforschung durch räumliche Konzentration zu verbessern. Die angestrebte Zentralisierung wissenschaftlicher Einrichtungen war damals nur dezentral, das heißt am Rande der Stadt 
realisierbar. Es war also gerade die Abkehr von der Stadt, die „Suburbanisierung von Wissenschaft", die für diese Projekte ausschlaggebend war. Im Falle des ehemaligen Bauerndorfs Garching waren es die Ansiedlung eines Forschungsreaktors in den fünfziger Jahren und die spätere Verlagerung verschiedener Hochschulinstitute der Technischen Universität, die den Weg zu einer Wissenschaftsstadt ebneten. In Martinsried wurde 1973 das MaxPlanck-Institut für Biochemie gegründet, um das sich in den folgenden Jahrzehnten andere Wissenschaftseinrichtungen gruppierten.

Seit den siebziger Jahren trat jedoch bei allen drei untersuchten Standorten das Bestreben in den Vordergrund, nicht mehr nur vorrangig die wissenschaftliche Forschung und Lehre, sondern die Umsetzung von Forschung in ökonomisch verwertbare Innovationen zu fördern. In diesen „Technopolen“ oder „kreativen Städten“, wie die Autorin diese in Abgrenzung von reinen Wissenschaftsstädten nennt, lösten sich die institutionellen und räumlichen Grenzen zwischen Wissenschaft und Ökonomie zusehends auf. So kam es in Garching und Martinsried zur Ansiedlung von Verwertungsgesellschaften und Unternehmen, die eng mit den Forschungsinstituten verbunden waren. Bei dem in den siebziger Jahren in der Trabantenstadt Neuperlach gegründeten Elekronikstandort handelte es sich von vornherein um eine Fortentwicklung der Forschungsabteilungen der Firma Siemens.

Die Autorin zeigt, wie sich diese veränderte Programmatik auch in einer neuen städtebaulichen Gestaltung niederschlug. Die Planungen für Garching (und teils auch von Martinsried) waren noch durch das „funktionalistischseparatistische Denken“ der städtebaulichen Moderne bestimmt. Entsprechend waren dort zunächst rein monofunktionale Forschungsansiedlungen entstanden, die kaum mit den bestehenden örtlichen Strukturen verbunden waren und die von Nutzern und Anwohnern als eintönig und gesichtslos empfundenen wurden. Demgegenüber war man seit den späten siebziger Jahren zunehmend bestrebt, den Forschungseinrichtungen durch eine bewusste Mischung von Funktionsbereichen und baulicher Verdichtung ein lebendiges urbanes Umfeld zu verschaffen. Hierzu gehörte auch die Anlage städtischer Gestaltungselemente wie öffentlicher Plätze oder Cafés, durch die spontane Begegnungen und Kommunikation zwischen den in den Forschungsstandorten arbeitenden Gruppen stimuliert werden sollten.

Ein zentrales Anliegen der Autorin besteht in der kritischen Auseinandersetzung mit der Idee der „kreativen Stadt“, die gegenwärtig in der Stadtforschung diskutiert wird und die auch zur Legitimation solcher Forschungsstandorte dient. Die aktive Förderung von Urbanität soll danach ein günstiges Umfeld für Kommunikation und Kreativität schaffen und dadurch die wirtschaftliche Innovationskraft eines Standortes stärken. Für die Autorin handelt es sich hierbei jedoch um einen vom Bild der antiken Polis inspirierten Mythos, der wenig mit der Realität der stets noch vorwiegend von Suburbanisierungsdynamiken bestimmten Forschungsstädte zu tun hat. 
Entsprechende planerische Bemühungen hätten daher allein zur Inszenierung einer leblosen postmodernen Scheinurbanität geführt. Urbanität und die mit ihr verbundenen Attribute der Kreativität und Spontanität ließen sich eben gerade nicht beliebig sozialtechnologisch herstellen.

So plausibel die Diagnose des Scheiterns der drei Forschungsstandorte als urbane Orte auch ist, so scheint das ihren Erfolg als Organisationsmodell der technologieorientierten Forschung jedoch kaum geschmälert zu haben. Die wissenschafts- und technikgeschichtliche Bedeutung des Buches liegt vor allem darin, durch die detaillierte Analyse exemplarischer Projekte die sozialräumlichen Transformationen und Konflikte sichtbar gemacht zu haben, die den Aufstieg dieses Modells begleitet haben.

Jens Lachmund (Maastricht)

Westermann, Andrea, 2007. Plastik und politische Kultur in Westdeutschland. Zürich: Chronos (=Interferenzen, 13. Studien zur Kulturgeschichte der Technik, hg. von David Gugerli), brosch. 387 S., CHF 58.00, ISBN-13: 978-3-0340-0849-5.

Monsieur Arpel verkörpert in Jacques Tatis Film „Mon Oncle“ das moderne, das neue, durchrationalisierte Frankreich der 1950er Jahre. Nicht zufällig ist der Protagonist dieser Welt ein Plastikfabrikant, dessen Unternehmen kilometerlange, farbige Plastikschläuche herstellt. Deren reibungslose Produktion gerät lediglich dann ins Chaotische, wenn Monsieur Hulot, der Repräsentant der altmodischen, vormodernen Welt, die Fabrik betritt. Plastik ist ein Stoff der Moderne, einer der Werkstoffe, der zu den, wie Andrea Westermann schreibt, „Materialien des 20. Jahrhunderts“ gehört. Dass sich diese Materialien eignen, um die Geschichte des 20. Jahrhunderts zu schreiben, machte bereits Jeffrey Meikle mit seinem 1995 publizierten Buch „American Plastic" deutlich, mit dem er eine Kulturgeschichte des Kunststoffs vorlegte. Für die Bundesrepublik fehlt Vergleichbares. In diese Lücke stößt Andrea Westermann mit ihrer Bielefelder Dissertation. Ihre Stoßrichtung ist jedoch eine andere. Sie konzentriert sich auf die Geschichte des PVC, „um nach dem Zusammenhang zwischen dem technisch-ökonomischen und dem gesellschaftlich-politischen Wandel Westdeutschlands zu fragen“ (S. 7). Ihr Buch versteht sie als einen technikhistorischen Beitrag zur Geschichte der Bundesrepublik. Sie schließt daher an Forschungen von Axel Schildt, Konrad Jarausch, Edgar Wolfrum und anderen an, die den Zusammenhang von erfolgreicher Marktwirtschaft und Demokratie herausgearbeitet haben. Angenommen werde, so fasst die Autorin zusammen, „ein Bedingungsverhältnis zwischen Demokratisierung und gesellschaftlicher Liberalisierung auf der einen Seite und marktwirtschaftlichem Erfolg auf der anderen Seite, dessen strukturelle Dynamik jedoch eher unterstellt als begründet wird“ 
(S. 8). Sie konstatiert, dass bislang wenig darüber bekannt sei, „wie sich dieser Wirkungszusammenhang methodisch und empirisch fassen lässt" (ebd.). Und genau hier möchte Westermann ansetzen. Ihr methodisches Herangehen orientiert sich dabei an Forschungen, die die politische und soziale Bedeutung von Dingen aufgezeigt haben (vor allem Appadurai, Callon/Latour). Eine ihrer zentralen Prämissen ist, dass „sich gesellschaftliche Beziehungen selten ohne Rückgriff auf Artefakte ergeben und auf Dauer stellen“ (S. 19). Vermeiden möchte sie jedoch strukturalistische Deutungen, weshalb sie zugleich an handlungstheoretische Überlegungen anschließt. Ziel ist es, die "artefaktfundierte Vergesellschaftung" in der Bundesrepublik nachzuzeichnen. PVC als wissenschaftlich-technisches Artefakt wird somit Bestandteil politischer Kultur.

Die Arbeit ist entlang der „Karriere des Werkstoffes PVC“ gegliedert und geht einerseits chronologisch vor, andererseits schildern die meisten Kapitel, entsprechend des handlungstheoretischen Ansatzes, Akteure und ihre Handlungsmuster. In Kapitel 1 und 2 analysiert Westermann die „Kunststoffakteure" wie Ingenieure, Wissenschaftler und Unternehmer. Sie rekonstruiert wissenschaftsgeschichtlich die Entwicklung des PVC, zeichnet die Umdeutungsbemühungen von einem Ersatzstoff zu einem funktionalen modernen Material nach und weist die „technikaffine Modernität" der Akteure als Teil der politischen Kultur aus. Weiter untersucht sie die sich formierende Branche der Kunststoffhersteller (Kapitel 2). In Kapitel 3 widmet sich die Autorin der Rolle von PVC in der Verbraucherdemokratie, ein Begriff, für den sich Westermann im Anschluss an angelsächsische Forschung bewusst entschieden hat. Sie konstatiert hier vor allem die entpolitisierende Integrationsfunktion von Kunststoff für eine massenkulturelle Vergesellschaftung, nimmt aber auch die technikkritischen Diskurse in den Blick. Im vierten Kapitel werden die Verbraucherproteste seit Ende der 1960er Jahre und damit die Repolitisierung der bundesrepublikanischen Kultur geschildert.

Westermanns Buch beeindruckt durch die souveräne Zusammenführung wissenschafts-, politik- und konsumgeschichtlicher Ansätze sowie die Einbettung in bundesrepublikanische Forschungen. Vor allem ist ihr artefaktorientierter Forschungsansatz hervorzuheben, der die unterschiedlichen Stränge gekonnt verknüpft. Neuere Forschungen zur Verwissenschaftlichung aufnehmend, strukturiert sie ihre Arbeit um ein wissenschaftlich-technisches Artefakt herum und zeigt dessen Bedeutung für die Entpolitisierung der bundesrepublikanischen politischen Kultur. Zudem zeigt sie anhand dieses Artefakts auch die Repolitisierung seit den 1960er Jahren und damit die Reinterpretation des Materials. Somit gelingt ihr ein innovativer Ansatz von technik- oder artefaktfundierter Gesellschaftsgeschichtsschreibung.

Gleichwohl bleibt Kunststoff im Buch über weite Strecken auf merkwürdige Weise abstrakt. Zwar betont Westermann die „strukturelle Dynamik“ zwischen einem Werkstoff und der politischen Kultur, indem sie die Rolle 
des Kunststoffs als Verpackung, für ein neues Warensortiment, neue Formen des Warenflusses, den Massenkonsum sowie schließlich als Objekt einer Protestbewegung aufzeigt. Doch bleibt das Artefakt selbst aufgrund der Entscheidung für die Untersuchung der Akteure über weite Strecken blass. Ausnahmen stellen die Passagen über Plastikhäuser und die Nutzung des Plastiks im öffentlichen Raum dar (Kapitel 3). Davon hätte man sich mehr gewünscht, denn es hätte die Spezifik des Artefakts für die Vergesellschaftungsprozesse schärfer hervortreten lassen. So scheinen viele der Ausführungen (vor allem in Kapitel 4) über weite Strecken auf andere Politikfelder übertragbar, und PVC gerät zuweilen nur mehr zum Stoff, der das Schreiben der bundesrepublikanischen Geschichte strukturiert, dessen Ästhetik, dessen Formen, dessen Produkte und dessen Nutzungen jedoch mehr Aufmerksamkeit verdient hätten. Gleiches gilt für die Konsumenten, die nur sehr vermittelt in den Blick geraten. Hier sei die kritische Nachfrage erlaubt, ob der Zusammenhang von politischer Kultur und Artefakt nicht unabdingbar des genaueren Blicks auf die Konsumenten bedarf. Gerade wenn Westermann in der Einleitung formuliert: „Die herrschende politische Kultur einer Gesellschaft muss durch die Analyse der alltäglichen sozialen Praktiken ihrer Mitglieder, beispielsweise im Umgang mit Kunststoff, beschrieben und erklärt werden“ (S. 15), hätte man sich die Betrachtung der alltäglichen Praktiken der Konsumenten gewünscht. Allerdings ist dies natürlich, wie gerade in der Technik- und Konsumgeschichte nur zu bekannt ist, nicht nur eine immer wieder gestellte Forderung, sondern auch eine schwierige Aufgabe. Aber vielleicht nimmt trotzdem jemand die Spur auf, die Andrea Westermannn hier so eindrucksvoll und überzeugend gelegt hat.

Martina Heßler (Offenbach)

\section{Dem Leben auf der Spur - Die Dauerausstellung des Berliner Medizinhistorischen Museums der Charité / Erweiterung und Neugestaltung 2006-2007}

Idee und Grundkonzept: Thomas Schnalke; Feinkonzept und Kuratoren: Isabel Atzl, Roland Helms, Beate Kunst, Petra Lennig, Thomas Schnalke, Navena Widulin; Gestaltung: Brigitte Fischer; Grafik: Christine Voigts, Hoch $3 \mathrm{GmbH}$, Patrick Kleinschmidt. http://www.bmm.charite.de/

Für das Berliner Medizinhistorische Museum der Charité hat sich in den letzten Jahren viel verändert. Der Backsteinbau liegt heute nicht mehr in einer verschwiegenen Ecke des Charité-Campus, sondern ist nun eine der zentralen Eingangspforten zu einem wissenschaftshistorisch wie wissenschaftlich bedeutsamen Gelände in Berlin-Mitte. Der neue Hauptbahnhof liegt unmittelbar gegenüber und bildet eine eindrucksvolle Kulisse für die Konzentration von Museumsgebäuden in diesem Teil Berlins: Das Naturhistorische 
Museum ist fünf Gehminuten entfernt und der Hamburger Bahnhof liegt in unmittelbarer Sichtweite. Mit der Nationalgalerie im Hamburger Bahnhof Museum der Gegenwart hat das Berliner Medizinhistorische Museum 2007 bereits gemeinsam die Ausstellung Schmerz verwirklicht. Seitdem konnten neue Besuchergruppen für die Häuser gewonnen werden und die Besuchszahlen im Medizinhistorischen Museum sind stark angestiegen.

$\mathrm{Zu}$ Recht, denn innen hat sich das Museum weit mehr noch als im Außenbereich gewandelt und professionalisiert. Wer heute das Haus besucht, findet eine Mischung von sensibler Umgestaltung und ursprünglicher Atmosphäre vor. Beispiel: Bis vor einiger Zeit wurde man im Museumsshop im Erdgeschoss durch einen Herrn in weißer Pflegerkleidung begrüßt. Man kann dies als eine Besonderheit des Museums vermissen, zugleich ist man aber schneller in der Lage, das Museum als eigenständiges Haus und nicht als Annex eines Klinikums zu sehen. Dagegen findet man im zweiten Stock immer noch die sogenannten Nasspräparate (in Konservierungsflüssigkeit aufbewahrte Präparate) in ihrer ursprünglichen Aufstellung und in den originalen beeindruckenden Vitrinen vor, durch einige erläuternde Schrifttafeln ergänzt.

Vor diesem Hintergrund einer schrittweisen und nicht bloß umkrempelnden Umgestaltung ist die Neueinrichtung der Dauerausstellung zu sehen. Während im ersten Stockwerk die jeweils immer über Eck liegenden Ausstellungssäle für Wechselausstellungen reserviert sind, folgen im zweiten und dritten Stockwerk die beiden Ebenen der Dauerausstellung, die über das zentrale Treppenhaus miteinander verbunden sind. Gemeinsame Gestaltungselemente halten die Stockwerke zusammen, wie zum Beispiel das Text-Bild-Band an der Wand, das in chronologischer Reihenfolge die Entwicklungsstationen der Charité als Klinik beschreibt. Dieses, durch die Jahreszahlen prominent erscheinende Band macht deutlich, dass im zweiten Stock die Frühzeit der Medizin und im dritten Stock vor allem das 19. Jahrhundert bis zur Gegenwart dargestellt werden.

Diese Aufteilung ist gut begründet: Frühe Kunst- und Wunderkammern, das Anatomische Theater und die Privatsammlungen bilden den Auftakt zum Verständnis für die Entwicklung der Medizin und die Entwicklung der Charité in Berlin. Beides ist geschickt miteinander verwoben und parallelisiert den wissenschaftshistorischen Überblick mit einer Institutionengeschichte. Die Darstellung der "Geburt der Klinik“ schließlich im selben Raum macht die historische Scheidung deutlich, die nicht nur die Medizin kennzeichnet: die Trennung in grundlegende Forschung einerseits und deren Anwendung in den Heilverfahren andererseits. Diese Aufteilung durchzieht den gesamten Ausstellungskomplex und wird mal in die eine, mal in die andere Richtung gewendet. Zentrales Ausstellungsstück und gleichzeitig die notwendige Überleitung in die Geschichte des Hauses auf diesem Stockwerk ist schließlich der originale Schreibtisch Rudolf Virchows, der direkt in den 
sich anschließenden langgestreckten Saal mit den großen Nasspräparate-Vitrinen führt. Arbeitsmöbel und Arbeitsergebnisse eines Mannes stehen hier im Vordergrund und verweisen auf den Gründer des Hauses. Die großartige historische Präparatesammlung, geordnet nach dreizehn anatomischen Oberbegriffen, ist an sich nichts Besonderes, diese Objekte gibt es auch an anderen Orten und man sieht sie heute mehr denn je ausgestellt. Das Besondere des Ortes aber ist - neben der Fülle der Objekte -, dass man spürt, dass diese Vitrinen mit ihrem Inhalt für den Raum konzipiert wurden, der Raum in Hinblick auf die Vitrinen proportioniert ist. Hat man diese beiden ersten Räume durchschritten, so ist bereits ein wesentlicher Teil des neuen Konzepts deutlich geworden: Es geht nicht um die Heroen der Medizin, es geht nicht um eine Institutionengeschichte allein, sondern alle Akteure der Vergangenheit kommen zu Wort und bewegen sich zwischen Charité, Berlin und allgemeiner Wissenschaftsentwicklung.

Dies wird besonders deutlich im darauf folgenden Stockwerk der neuen Präsentation. Hier bestimmen zwei Bilder die ineinander übergehenden Säle: das Labor und der Patientensaal. Der erste Raum beginnt mit der naturwissenschaftlich begründeten Medizin in der ersten Hälfte des 19. Jahrhunderts. Die Vorstellung des Körpers als eine Art physiologische Maschine wird verdeutlicht und leitet über zur Laborwissenschaft, wie sie unter anderem in Berlin begründet wurde. Das „Labor“ gibt etwa Auskunft über die Geschichte der Bakteriologie und endet mit den zeitgenössisch relevanten Themen wie der Entzifferung des Genoms oder der Hirnforschung. In diesem Raum wird das Potential des historischen Ortes in besonderer Weise herausgearbeitet: Während alle Fenster des Gebäudes mit Gazebildern verhangen sind, die zwar einen Durchblick ermöglichen, aber zugleich die angesprochenen Raumbilder thematisieren, fällt der Blick in der Abteilung der Medizin an der Charité im Nationalsozialismus unverhüllt auf das Außengelände des Klinikums und seine Bauten. Ebenfalls von diesem Raum aus, dem "Labor", blickt man am Ende von oben in die angrenzende und durch das Museum genutzte Hörsaalruine, in der Virchow und andere lehrten und die eindrucksvoll die Authentizität des Ortes verstärkt.

Der letzte langgestreckte Raum ist schließlich dem Patienten gewidmet. Gleich einem Krankensaal um 1900 sind rechts und links vom Mittelgang „Betten“ aufgestellt, Vitrinenkonstellationen, die Fußende, Matratze und Kopfstütze nachempfinden. Hier werden jeweils ein Patient/eine Patientin durch seine oder ihre Krankengeschichte vorgestellt, und entsprechende historische Objekte verweisen auf die medizinische Versorgung und die Behandlungsmethoden der Zeit. Dieser Raum zitiert die Aufteilung des direkt darunterliegenden, in dem die Nasspräparate ausgestellt sind. Wo aber die Präparate auf ein „dreidimensionales Körperinventar“ verweisen, ist hier die Umsetzung medizinischer Forschung in einen exemplarischen Krankheitsverlauf und eine biographische Zuspitzung erfolgt. 
Dem Leben auf der Spur stellt die in Teilen naturwissenschaftlich gefasste Medizin der letzten drei Jahrhunderte vor und zitiert mit diesem Titel eine der leitenden Handlungsmaximen des Berliner Arztes, Wissenschaftlers und Politikers Rudolf Virchow. Dieser eröffnete in dem Backsteinbau 1899 das „Pathologische Museum“ mit Tausenden von Nasspräparaten. Für eine hier anknüpfende sammlungs- und museumsgeschichtliche Perspektive hätte man sich etwas mehr Raum gewünscht, mit einigen, auch durch die Gestaltung der Ausstellung hervorgehobenen Elementen. Es leuchtet sofort ein, dass mit dem neuen Konzept nicht nur einfach die Berliner Erfolgsgeschichte von Helmholtz über Virchow bis heute dargestellt werden sollte. Das ist auch gelungen! Doch einige grundlegende Daten mehr, einige von den hinreißenden alten Fotografien der ursprünglichen Räume und ihrer Einrichtung hätten geholfen, den so bedeutsamen Zusammenhang von Ort und Objekten dem Besucher vor Augen zu führen. Denn hierin liegt das Faszinierende einer heute immer noch zugänglichen Sammlung, die ursprünglich einem rein medizinischen Lehrzusammenhang zu verdanken war: In ihr überkreuzen sich verschiedene Zeitebenen. Ist sie für die einen immer noch Teil eines Ausbildungsganges (etwa für Krankenpflegeschüler), stellt sie für die anderen eine kulturwissenschaftlich geöffnete Annäherung an ein wissenschaftliches Thema dar. Dem Leben auf der Spur leistet einen anschaulichen und übersichtlich geordneten Rundgang durch die Medizingeschichte. Die erklärenden Texte sind zweisprachig, nie zu lang und immer leicht verständlich und eingängig. Die Gestaltung ist zurückhaltend mit kleinen inszenatorischen Elementen versehen. Leider können die aufgerufenen, leitenden Raumbilder nicht immer klar und konsequent umgesetzt werden. Der Betrachter vermag sie zwar an Details oder einzelnen Hinweisen erahnen (zum Beispiel mit Hilfe der erwähnten Gazebilder an den Fenstern), aber eine eindeutige räumliche Lösung für ihre konzeptionell einleuchtende Abfolge fehlt an einigen Stellen.

Das zur neuen Ausstellung gehörige Plakat zeigt ein Augenmodell aus der zweiten Hälfte des 19. Jahrhunderts, das - so ist dem Objektschild zu dem auch in der Ausstellung gezeigten wunderbaren Modell zu entnehmen - den „Verlauf und den Einfluß der zwölf Augenmuskeln auf die Stellung des Augapfels“ verdeutlicht. Das Auge als ein im Modell umgesetztes Untersuchungsobjekt der Wissenschaften und das Auge als ironisierendes Sujet der irritierenden Frage, wer auf wen schaut, fasst den Museumsbesuch der neuen und äußerst sehenswerten Dauerausstellung im Berliner Medizinhistorischen Museum präzise zusammen.

Anke te Heesen (Tübingen) 


\title{
Zur Publikation in NTM angenommene Beiträge
}

\author{
Alexander von Schwerin: Prekäre Stoffe. Radiumökonomie, \\ Risikoepisteme und die Etablierung der \\ Radioindikatortechnik in der Zeit des \\ Nationalsozialismus
}

Anne Cottebrune:

Zwischen Theorie und Deutung der Vererbung psychischer Störungen. Zur Übertragung des Mendelismus auf die Psychiatrie in Deutschland und in den USA, ca. 1911-1930

Heiko Stoff:

Hexa-Sabbat. Fremdstoffe und Vitalstoffe, Experten und der kritische Verbraucher in den 1950er und 60er Jahren 